\title{
THE BRAUER GROUP OF GRADED AZUMAYA ALGEBRAS. II: GRADED GALOIS EXTENSIONS $\left({ }^{1}\right)$
}

\author{
BY
}

\author{
LINDSAY N. CHILDS
}

\begin{abstract}
This paper continues the study of the Brauer group $B_{\phi}(R, G)$ of $G$-graded Azumaya $R$-algebras begun in [5]. A group $\operatorname{Galz}_{\phi}(R, G)$ of graded Galois extensions is constructed which always contains, and often equals, the cokernel of $B_{\phi}(R, G)$ modulo the usual Brauer group of $R$. Sufficient conditions for equality are found. The structure of $\operatorname{Galz}_{\phi}(R, G)$ is studied, and $\operatorname{Galz}_{\phi}\left(R,\left(Z / p^{e} Z\right)^{r}\right)$ is computed. These results are applied to give computations of a Brauer group of dimodule algebras constructed by F. W. Long.
\end{abstract}

In the first paper of this name ([5] $=[C G O])$ we introduced and studied a Brauer group of graded Azumaya algebras $B_{\phi}(R, G)$ for $G$ a finite abelian group, $R$ a commutative ring with units group $U(R)$, and $\phi: G \times G \rightarrow$ $U(R)$ a fixed bimultiplicative map. When $G=Z_{2}$ and $\phi$ is nontrivial, $B_{\phi}(R, G)$ is the Brauer-Wall group ([19], [3], [17]), introduced to provide an appropriate image for the Clifford algebra map on quadratic spaces. When $\phi$ is trivial, $B(R, G)$ was studied in [14].

This paper is a sequel to [CGO] and is motivated by two problems arising in [CGO]. One problem was that of finding an appropriate group of graded Galois extensions into which $B_{\phi}(R, G)$ is mapped (via a map called $\pi$ in [CGO]) with kernel $B(R)$, the usual Brauer group. In [CGO] this was solved by an ad hoc method of inducing a product on the image of $\pi$ from that on $B_{\phi}(R, G)$, and computing this product explicitly as needed. This sufficed to describe $B_{\phi}(R, G)$ for $G$ cyclic. In this paper we remedy the ad hoc treatment of the image of $\pi$ by introducing $(\S 1)$ a group $\operatorname{Galz}_{\phi}(R, G)$ of graded Galois extensions, with the group structure intrinsically defined, into which $\pi$ maps as a homomorphism. It turns out that the group $\operatorname{Galz}_{\phi}(R, G)$ can be described rather explicitly in many cases, and this leads to progress on the second problem left over from [CGO], namely, describing $B_{\phi}(R, G)$ when $G$ is noncyclic.

Received by the editors September 27, 1973.

AMS (MOS) subject classifications. Primary 16A16.

(1) Research partially supported by the National Science Foundation Grant GU 3171 and Grant PO 29652. 
Suppose, as we shall from $\S 3$ on, that $R$ is a connected commutative ring, $G$ is an abelian group of order $n$ and exponent $m$, and $R$ contains $1 / n$ and a primitive $m$ th root of unity. We show in $\S 2$ that to compute $\operatorname{Galz}_{\phi}(R, G)$ it suffices to do it for $G$ a $p$-group. In $\S 3$ we compute $\operatorname{Galz}_{\phi}(R, G)$ when $G=$ $Z_{p e^{e}} \times \cdots \times Z_{p e}(r$ copies) in terms of $\operatorname{Comm}(R, G)$, the group of commutative Galois extensions with group $G$ and in terms of two $r \times r$ matrix groups with entries in $Z / p^{e} Z$ : a group of skew matrices, and an orthogonal group $O^{r}(M)$. If $p$ is odd and $\phi_{s}: G \times G \rightarrow U(R)$, defined by $\phi_{s}(\sigma, \tau)=\phi(\sigma, \tau), \phi(\tau, \sigma)$, is nondegenerate, our description becomes the exact sequence

$$
1 \rightarrow \operatorname{Comm}(R, G) \rightarrow \operatorname{Galz}_{\phi}(R, G) \rightarrow O_{Z / p}^{r} z^{(M)} \rightarrow 1
$$

where $M$ is a nonsingular symmetric matrix representing $\phi_{s}$.

In $\S 4$ we show that, with $R, G$ as above, if $\operatorname{Pic}(R)$ is $n$-torsion free, then the map $\pi$ maps onto $\operatorname{Galz}_{\phi}(R, G)$, thereby providing a description of $B_{\phi}(R, G)$ for a large class of noncyclic groups.

In [12], [13], F. W. Long, motivated by the theory of equivariant Brauer groups of Fröhlich and Wall [9], constructed a Brauer group $B D(R, H)$ of dimodule algebras for any finite commutative, cocommutative Hopf algebra $H$. We observe ( $\S 5)$ that when $H=R G$ and $R, G$ are as described above, $B D(R, H) \cong B_{\phi}(R, G \times G)^{\circ}$ for an appropriate $\phi$. Thus the results in this paper provide a description of Long's group, whenever $R, G$ and $\operatorname{Pic}(R)$ are as described above and $G=\Pi_{p} G_{p}$ with $G_{p}=Z_{p} e \times \times Z_{p e}$ ( $n$ times) for any $n$ and $e$, in terms of $B(R), \operatorname{Comm}(R, G)$ and $O_{Z / p^{e} Z}^{2 n^{p e}}\left(\begin{array}{ll}0 & I \\ I & 0\end{array}\right)$.

Assume throughout the paper that $R$ is a commutative ring, $G$ is a finite abelian group, and $\phi: G \times G \rightarrow U(R)$ is a bilinear map. Recall that if $A=$ $\Sigma_{\sigma \in G} A_{\sigma}, h A=\bigcup_{\sigma \in G} A_{\sigma}$. A formula which only makes sense for elements in $h A$ is assumed to be valid as shown only where it makes sense and is extended by linearity on all of $A$. The $\phi$ in $B_{\phi}(R, G)$, etc., will often be omitted. Boldface $\otimes$ denotes the graded tensor product of [CGO]. The product on $G$ will be written multiplicatively, with identity 1 .

All unexplained notation is from [CGO].

1. The group of graded Galois extensions. Here is the group of graded Galois extensions which is a candidate for an intrinsic description of the image of the map $\pi$ of [CGO], and is, in any case, a target for $\pi$ :

(1.1) Definition. Let $\operatorname{Galz}_{\phi}(R, G)$ be the set of isomorphism classes (where isomorphisms preserve all structure) of $R$-algebras $S$ with the following structures:

(i) $S$ is a $G$-graded $R$-algebra. 
(ii) $S$ has a right $G$-action with $G$ acting as grade-preserving $R$-algebra automorphisms.

(iii) $S$ has a left $G$-action with $G$ acting as grade-preserving $R$-algebra automorphisms.

These structures satisfy the following axioms, which are not independent:

(1) $S$ is a graded Azumaya $S_{1}$-algebra $\left(S_{1}=\right.$ trivially graded part of $S$, as in [CGO]).

(2r) $S$ is a Galois extension of $R$ on the right with group $G$.

(2l) $S$ is a Galois extension of $R$ on the left with group $G$.

(3r) For all $a, b$ in $h S, b a^{b}=\phi(b, a) a b$, where if $b$ is in $S_{\sigma}, a^{b}=a^{\sigma}$ denotes the right action of $\sigma$ on $a$.

(3i) For all $a, b$ in $h S, a b=\phi(a, b) b\left({ }^{b} a\right)$, where ${ }^{b} a$ denotes left action.

(4) For all $a$ in $S, \sigma$ in $G, a^{\sigma}=\phi(\sigma, a) \phi(a, \sigma)^{\sigma} a$.

It is easy to see that $(3 r)+(4)$ implies $(3 l)$, and that $(3 r)$ implies the right center of $S$ is $S_{1},(3 l)$ implies the left center of $S$ is $S_{1}$, and (2) implies separability of $S$ over $S_{1}$, so $(3 r)+(3 l)+(2)$ implies (1).

Recall that a graded Azumaya $R$-algebra $A$ is called fully graded [CGO, p. 309] if, for all $\sigma, \tau$ in $G, A_{\sigma} A_{\tau}=A_{\sigma \tau}$. Each class in the Brauer group $B(R, G)$ has a fully graded representative. For a fully graded Azumaya $R$-algebra $A, \pi(A)=A^{A_{1}}=\left\{x\right.$ in $A \mid x a=a x$ for all $a$ in $\left.A_{1}\right\}$.

In the rest of this section we prove:

(1.2) TheOREM. The set $\operatorname{Galz}(R, G)$ is a group, the map $\pi$ is a homomorphism into $\operatorname{Galz}(R, G)$, and hence (by $[\mathrm{CGO},(3.13)]$ ) there is an exact sequence

$$
0 \rightarrow B(R) \rightarrow B(R, G) \rightarrow \operatorname{Galz}(R, G) .
$$

Proof. We begin by showing that for a fully graded Azumaya $R$-algebra $A, \pi(A)$ is an element of $\operatorname{Galz}(R, G)$.

The grading on $\pi(A)$ is that induced from the inclusion $\pi(A) \subseteq A$.

The right action of $G$ on $\pi(A)$ was described in [CGO, (p. 311)]: if $b_{\sigma}^{i}$ in $A_{\sigma^{-1}}, c_{\sigma}^{i}$ in $A_{\sigma}$ are elements satisfying $\Sigma_{i} b_{\sigma}^{i} c_{\sigma}^{i}=1$, then defining for $a$ in $A^{A}, a^{\sigma}=\phi(\sigma, a) \Sigma_{i} b_{\sigma}^{i} a c_{\sigma}^{i}$, we showed [CGO, (3.4)] that this action makes $\pi(A)$ into a Galois extension of $R$ with group $G$. From the definition it is clear that the right action of $G$ preserves the grading by $G$ on $\pi(A)$. We also showed [CGO, (3.3)] that

$$
\pi(A) \subseteq\left\{a \text { in } A \mid x a^{x}=\phi(x, a) a x \text { for all } x \text { in } h A\right\} ;
$$

the converse is obvious. Thus $\pi(A)$ satisfies (3r) and $(2 r)$ for the right $G$-structure. 
140

L. N. CHILD

The left $G$-structure arises from the fact that if $A$ is a graded Azumaya $R$-algebra, so is $A^{\#}$, and $\pi\left(A^{\#}\right)=(\pi(A))^{\#}$ is also a graded $R$-algebra with a right $G$-structure. The grading on $A^{\#}$ is the same as that on $A$.

Define the left $G$-structure on $\pi A$ by $\left({ }^{\sigma} a\right)^{\#}=\left(a^{\#}\right)^{\sigma^{-1}}$. Then, since $\pi(A)^{\#}$ satisfies (ar), $\pi(A)$ satisfies (2l). Since $\pi(A)^{\#}=\left\{a^{\#} \mid x^{\#} a^{\# x}=\phi(x, a) a^{\#} x^{\#}\right.$ for all $x$ in $h A\}$, therefore (recalling that $\left.x^{\#} a^{\#}=\phi(x, a)(a x)^{\#}\right)$

$$
\pi(A)=\left\{a \mid a x=\phi(a, x) x\left({ }^{x} a\right) \text { for all } x \text { in } h A\right\},
$$

hence satisfies (Bl).

If $d_{\sigma}^{i} \in A_{\sigma}, e_{\sigma}^{i} \in A_{\sigma^{-1}}, \Sigma d_{\sigma}^{i \#} e_{\sigma}^{i}=\Sigma e_{\sigma}^{i} d_{\sigma}^{i} \phi\left(\sigma, \sigma^{-1}\right)=1$, then

$$
\begin{aligned}
\left({ }^{\sigma} a\right)^{\#}=\left(a^{\#}\right)^{\sigma^{-1}} & =\phi\left(\sigma^{-1}, a\right) \sum d_{\sigma}^{i \#} a^{\#} e_{\sigma}^{i \#} \\
& =\phi\left(\sigma^{-1}, a\right) \sum \phi\left(d_{\sigma}^{i}, a\right) \phi\left(d_{\sigma}^{i} a, e_{\sigma}^{i}\right)\left(e_{\sigma}^{i} a d_{\sigma}^{i}\right)^{\#}
\end{aligned}
$$

so

$$
\sigma_{a}=\phi\left(\sigma^{-1}, a\right) \phi(\sigma, a)\left(\sigma a, \sigma^{-1}\right) \sum e_{\sigma}^{i} a d_{\sigma}^{i},
$$

but $a^{\sigma}=\phi(\sigma, a) \Sigma e_{\sigma}^{i} a\left(d_{\sigma}^{i} \phi\left(\sigma, \sigma^{-1}\right)\right)$ so $\sigma_{a}=\phi\left(\sigma^{-1}, a\right) \phi\left(a, \sigma^{-1}\right) a^{\sigma}$, verifying (4). Thus $\pi(A)$ satisfies all the axioms.

The product on $\operatorname{Galz}(R, G)$ will be motivated by that induced by $\pi$, which we now describe. For $A, B$ in $B(R, G)$,

$$
\begin{aligned}
\pi(A \otimes B)=\left\{\sum a_{i}\right. & \otimes b_{i} \mid\left(\sum a_{i} \otimes b_{i}\right)(x \otimes y) \\
& \left.=\sum(x \otimes y)\left(a_{i} \otimes b_{i}\right) \text { for all } x \otimes y \text { in } A_{\sigma} \otimes B_{\sigma^{-1}}\right\} .
\end{aligned}
$$

Since $\pi(A \otimes B) \subseteq \pi(A) \otimes \pi(B)$,

$$
\begin{aligned}
& \left(\sum a_{i} \otimes b_{i}\right)(x \otimes y)=\sum(x \otimes y)\left(a_{i} \otimes b_{i}\right)=\sum \phi\left(y, a_{i}\right) x a_{i} \otimes y b_{i} \\
& \quad=\sum \phi\left(b_{i}, x\right) a_{i} x \otimes b_{i} y=\sum \phi\left(b_{i}, x\right) \phi\left(x, a_{i}\right)^{-1} x a_{i}^{x} \otimes \phi\left(b_{i}, y\right) y^{y} b_{i} .
\end{aligned}
$$

Since $\phi(\sigma, x y)=1=\phi(x y, \sigma)$ for all $\sigma$, it follows that $\Sigma x a_{i}^{x} \otimes y^{y} b_{i}=$ $\Sigma x a_{i} \otimes y b_{i}$, so $(x \otimes y)\left(\Sigma a_{i}^{\sigma} \otimes^{\sigma^{-1}} b_{i}-\Sigma a_{i} \otimes b_{i}\right)=0$ for all $x \otimes y$ in $A_{\sigma}$ $\otimes A_{\sigma^{-1}}$. Choose $z_{j}, w_{j}$ in $A_{\sigma^{-1}}, A_{\sigma}$, with $\Sigma z_{j} w_{j}=1$, and $x_{k}, y_{k}$ in $A_{\sigma}$, $A_{\sigma^{-1}}$ with $\Sigma x_{k} y_{k}=1$; then

$$
\begin{aligned}
0 & =\sum_{j, k}\left(z_{j} \otimes x_{k}\right)\left(w_{j} \otimes y_{k}\right)\left(\sum a_{i}^{\sigma} \otimes \sigma^{-1} b_{i}-\sum a_{i} \otimes b_{i}\right) \\
& =\sum a_{i}^{\sigma} \otimes^{\sigma^{-1}} b_{i}-\sum a_{i} \otimes b_{i} .
\end{aligned}
$$


Thus $\pi(A \otimes B)=\left\{\Sigma a_{i} \otimes b_{i} \mid \Sigma a_{i}^{\sigma} \otimes \sigma^{-1} b_{i}=\Sigma a_{i} \otimes b_{i}\right.$ for all $\sigma$ in $\left.G\right\}$. So we define $S \cdot T=\left\{\Sigma s_{i} \otimes t_{i}\right.$ in $S \otimes T \mid \Sigma s_{i}^{\sigma} \otimes \sigma^{-1} t_{i}=\Sigma s_{i} \otimes t_{i}$ for all $\sigma$ in $\left.G\right\}$.

The identity of $\operatorname{Galz}(R, G)$ is the image of the identity of $B(R, G)$, namely $G R=$ the set of all functions from $G$ to $R$ with pointwise addition and multiplication and with trivial grading [CGO, (3.7)]. The inverse of $S$ in $\operatorname{Calz}(R, G)$ is $S^{\#}$, with grading induced from $S$ and left and right actions by $G$ given by $\left(s^{\#}\right)^{\sigma}=\left(\sigma^{-1} s\right)^{\#},{ }^{\sigma}\left(s^{\#}\right)=\left(s^{\sigma^{-1}}\right)^{\#}$ [CGO, p. 314].

We must show that $\operatorname{Galz}(R, G)$ is closed under multiplication and that the axioms for a group hold. Once we do that it will be clear that $\pi$ is a homomorphism and, given [CGO, §3], the theorem will be proved.

Closure. The right action on $\pi(A \otimes B)$ is induced from the right action on $B$ : let $c_{\sigma}^{i} \in B_{\sigma^{-1}}, d_{\sigma}^{i} \in B_{\sigma}$ with $\Sigma c_{\sigma}^{i} d_{\sigma}^{i}=1$. Then

$$
\begin{aligned}
(a \otimes b)^{\sigma} & =\phi(\sigma, a b) \sum\left(1 \otimes c_{\sigma}^{i}\right)(a \otimes b)\left(1 \otimes d_{\sigma}^{i}\right) \\
& =\phi(\sigma, a b) \phi\left(\sigma^{-1}, a\right) \sum a \otimes c_{\sigma}^{i} b d_{\sigma}^{i} \\
& =\sum a \otimes \phi(\sigma, b) c_{\sigma}^{i} b d_{\sigma}^{i}=a \otimes b^{\sigma} .
\end{aligned}
$$

So for $S, T$ in $\operatorname{Galz}_{\phi}(R, G)$ define the right action on $S \cdot T$ to be that on $T$. Similarly, the left action on $\pi(A \otimes B)$ is induced from the left action on $A$, so define the left action of $G$ on $S \cdot T$ to be that on $S$.

Give $S \cdot T$ the grading induced from the product grading on $S \otimes T$. To check that $S \cdot T$ is in $\operatorname{Galz}(R, G)$ it suffices to show that

2. $S \cdot T$ is a Galois extension of $R$ on the right with group $G$ and on the left with group $G$, both of which preserve grading;

3. for all $a, b$ in $h(S \cdot T), b a^{b}=\phi(b, a) a b$;

4. for all $a$ in $h(S \cdot T), \sigma$ in $G, a^{\sigma}=\phi(\sigma, a) \phi(a, \sigma)^{\sigma} a$.

Proof of 2. If $S, T$ are in $\operatorname{Galz}(R, G)$ then $S \otimes T$ is a Galois extension of $R$ with group $G \times G,(\sigma, \tau)(s \otimes t)=s^{\sigma} \otimes{ }^{\tau} t$. For if $\left\{s_{i}, r_{i}\right\}$ are Galois elements for the right action of $G$ on $S$ (i.e., $\Sigma_{i} s_{i} r_{i}^{\sigma}=\delta_{1, \sigma}$ for all $\sigma \in G)$ and $\left\{t_{j}, u_{j}\right\}$ are Galois elements for the left action of $G$ on $T$, then it is quickly checked that

$$
\left\{s_{i} \otimes t_{j}, \phi^{-1}\left(t_{j}, r_{i}\right) r_{i} \otimes u_{j}\right\}
$$

is a set of Galois elements for $S \otimes T$; hence condition 1.3b of [4] holds for the action of $G \times G$ on $S \otimes T$. It follows easily by [4,2.2] that $S \otimes T$ is a Galois extension of $S \cdot T$ with group $D=\left\{\left(\sigma, \sigma^{-1}\right) \in G \times G\right\}$, and, hence, by $[4,1.6]$, that there exists an element $c=\Sigma_{k} c_{k} \otimes d_{k}$ in $S \otimes T$ with $\Sigma_{\sigma \in G} \Sigma_{k} c_{k}^{\sigma} \otimes \sigma^{-1} d_{k}=1 \otimes 1$, i.e., whose trace is one. By taking homogeneous components we can choose $c$ to be in $S_{1} \otimes T_{1}$. 
Then, following $\left[4\right.$, p. 23], let $\left\{v_{j}, w_{j}\right\}$ be Galois elements for the right action of $G$ on $T$, and $\left\{s_{i}, r_{i}\right\}$ be as above. Consider $\left\{x_{i j}, y_{i j}\right\}$ in $S \cdot T$ as follows:

$$
\begin{aligned}
& x_{i j}=\sum_{\sigma}\left(\left(s_{i}^{\sigma} \otimes^{\sigma^{-1}} v_{j}\right)\left(\sum_{k} c_{k}^{\sigma} \otimes^{\sigma^{-1}} d_{k}\right)\right), \\
& y_{i j}=\sum_{\sigma} \phi^{-1}\left(v_{j}, r_{i}\right)\left(r_{i}^{\sigma} \otimes^{\sigma^{-1}} w_{j}\right) .
\end{aligned}
$$

Each of these is the trace over $D$ of an element in $S \otimes T$ so is in $S \cdot T$. It is easily verified that $\left\{x_{i j}, y_{i j}\right\}$ are Galois elements for the right action of $G$ on $S \otimes T$; hence (2r) holds.

A similar argument holds to show (2l).

3. Let $a \otimes b, c \otimes d$ be homogeneous elements of $S \cdot T \subseteq S \otimes T$. Then $\phi(a b, c d)(c \otimes d)(a \otimes b)=\phi(a b, c d) \dot{\phi(d, a) c a} \otimes d b$

$$
\begin{aligned}
& =\phi(a b, c d) \phi(d, a) a c^{a} \phi(a, c)^{-1} \otimes b d^{b} \phi(b, d)^{-1} \\
& =\phi(b, c) \phi(a, d) \phi(d, a) a c^{a} \otimes b d^{b} \\
& =\phi(a, d) \phi(d, a)(a \otimes b)\left(c^{a} \otimes d^{b}\right)=\phi(a, d) \phi(d, a)(a \otimes b)\left(c \otimes^{a} d^{b}\right) \\
& =(a \otimes b)\left(c \otimes d^{a b}\right)=(a \otimes b)(c \otimes d)^{(a \otimes b)}
\end{aligned}
$$

and similarly for homogeneous elements of $S \cdot T$ which are sums of tensors in $S \otimes T$.

4.

$$
\begin{aligned}
(a \otimes b)^{\sigma} & =a \otimes b^{\sigma}=\phi(\sigma, b) \phi(b, \sigma) a \otimes^{\sigma} b \\
& =\phi(\sigma, b) \phi(b, \sigma) \phi(a, \sigma) \phi(\sigma, a)^{\sigma} a \otimes b \\
& =\phi(\sigma, a b) \phi(a b, \sigma)^{\sigma}(a \otimes b)
\end{aligned}
$$

and similarly for sums of tensors.

So $\operatorname{Galz}(R, G)$ is closed under multiplication.

Associativity. We have $S(T U)=\left(S \otimes(T \otimes U)^{D}\right)^{D}$. Since $T \otimes U$ is a Galois extension of $(T \otimes U)^{D}$ with group $D$, every element of $(T \otimes U)^{D}$ is the trace of some element of $T \otimes U$, etc. Thus a typical element of $S(T U)$ is of the form

$$
\sum_{i} \sum_{\sigma} s_{i}^{\sigma} \otimes^{\sigma^{-1}}\left(\sum_{j} \sum_{\tau} t_{i j}^{\tau} \otimes \tau^{-1} u_{i j}\right)=\sum_{i j} \sum_{\tau}\left(\sum_{\sigma} s_{i}^{\sigma} \otimes^{\sigma^{-1}} t_{i j}\right)^{\tau} \otimes^{\tau^{-1}} u_{i j} \text {, }
$$

a sum of elements in $\left((S \otimes T)^{D} \otimes U\right)^{D}=(S T) U$. 
Identity. The identity of $\operatorname{Galz}(R, G)$ is $G R=$ functions from $G$ to $R$ with trivial grading. On $G R$ the left and right $G$-structures coincide, and if $S$ is any graded Galois extension $S \otimes G R=S \otimes G R$ since $G R$ is trivially graded. Thus as a graded algebra and right Galois extension $S \cdot G R=S$ by the standard argument [CGO, p. 313]; since the left $G$-structure on $S \cdot G R$ is the same as that on $S, G R$ is the identity.

Inverse. The inverse of $S$ is $S^{\#}$, as described above. Consider the map $f:\left(S \otimes S^{\#}\right)^{D} \rightarrow G R$, defined by linearity and $f\left(x y^{\#}\right)(\tau)=x\left({ }^{\tau} y\right)$. The image of $f\left(x y^{\#}\right)$ is in fact in $R$ since

$$
\begin{aligned}
\left(x^{\tau} y\right)^{\sigma} & =x^{\sigma} \cdot \tau^{\sigma}=f\left(x^{\sigma} \otimes\left(y^{\sigma}\right)^{\#}\right)(\tau) \\
& =f\left(x^{\sigma} \otimes \sigma^{-1}\left(y^{\#}\right)\right)(\tau)=f\left(x \otimes y^{\#}\right)(\tau)=x^{\tau} y
\end{aligned}
$$

$f$ is an algebra map since

$$
\begin{aligned}
f(x & \left.\otimes y^{\#}\right)\left(z \otimes w^{\#}\right)(\tau)=f\left(x z \otimes y^{\#} w^{\#} \phi(y, z)\right)(\tau) \\
& =f\left(x z \otimes \phi(y, w)(w y)^{\#} \phi(y, z)\right)(\tau) \\
& =f\left(x z \otimes(w y)^{\#}\right)(\tau) \text { since } z w \text { is in } R \quad\left(f\left(z \otimes w^{\#}\right)(1)=z w\right) \\
& =x z^{\tau}(w y)=x z^{\tau} w^{\tau} y \\
& =\left(x^{\tau} y\right)\left(z^{\tau} w\right) \quad \text { since } z^{\tau} w \text { is in } R \\
& =f\left(x \otimes y^{\#}\right)(\tau) \cdot f\left(z \otimes w^{\#}\right)(\tau) .
\end{aligned}
$$

By $\left[10\right.$, Corollary 2, p. 5], it follows that $\left(S \otimes S^{\#}\right)^{D}=G R$, and $S^{\#}$ is the inverse of $S$ in $\operatorname{Galz}(R, G)$. This completes the proof.

2. Reduction to $p$-groups. The next two sections are devoted to a study of $\operatorname{Galz}_{\phi}(R, G)$ for a given fixed bilinear map $\phi: G \times G \rightarrow U(R)$. In this section we prove

(2.1) Theorem. Let $G=H \times J$ where the orders of $H$ and $J$ are relatively prime. Let $\phi_{H}, \phi_{J}$ be the restrictions of $\phi$ to $H, J$. Then $\operatorname{Galz}_{\phi}(R, G)=\operatorname{Galz}_{\phi_{H}}(R, H) \times \mathrm{Galz}_{\phi_{J}}(R, J)$.

(2.2) Corollary. Let $G=\Pi G_{p}$ be the decomposition of $G$ into its p-primary components, and let $\phi_{p}$ be $\phi$ on $G_{p} \times G_{p}$. Then

$$
\operatorname{Galz}_{\phi}(R, G)=\prod_{p} \operatorname{Galz}_{\phi_{p}}\left(R, G_{p}\right) .
$$

The equality of (2.1) will be obtained by decomposing any $S$ in 
$\mathrm{Galz}_{\phi}(R, G)$ as

$$
S \cong S^{J} \otimes_{R} S^{H}
$$

To prove (2.1) it suffices to show

(2.4) The decomposition (2.3) is as $R$-algebras and $G$-modules. respectively.

(2.5) The algebras $S^{J}$ and $S^{H}$ are in $\operatorname{Galz}_{\phi_{H}}(R, H), \mathrm{Galz}_{\phi_{J}}(R, J)$,

(2.6) The decomposition (2.3) gets along with the group structures on $\mathrm{Galz}_{\phi}(R, G)$, etc.

Proof of (2.4). Since $S$ is a Galois extension of $R$ with group $G=$ $H \times J$ it is standard that $S \cong S^{J} \otimes_{R} S^{H}$ as $R$-modules and $G$-modules. The only nontrivial aspect of (2.4) is in showing that (2.3) is a decomposition as $R$-algebras, that is, that elements of $S^{J}$ and $S^{H}$ commute with each other. To do this we look at the $R$-module generated by $\left\{x y-y x \mid x \in S^{J}, y \in S^{H}\right\}$ $\subseteq S$. To show it is zero and (2.4) holds it suffices to show it when we replace $R$ by a faithfully flat extension, namely, the direct sum of the stalks on the Boolean spectrum of $R$, a faithfully flat extension of $R[18,2.9]$. Since these stalks are connected $[18,2.13]$ it suffices to assume $R$ is connected. In that case, $S$, being a Galois extension of $R$ with abelian group $G$, is a central Galois extension of $T$, the center of $S$, with group $L$ [11, Proposition 8]. Then $S=$ $\Sigma \bigoplus J_{\sigma}$ with $J_{1}=T, J_{\sigma}$ rank one projective $T$ modules, for $\sigma$ in $L$ [11]. Here $J_{\sigma}=\left\{a \in S \mid a x^{\sigma}=x a\right\}$. The noncommutativity of $S$ is expressed by a skew nondegenerate bilinear form $\psi$ on $L$ : for $a$ in $J_{\sigma}, b$ in $J_{\tau}, b a=\psi(\tau, \sigma) a b$ [8], [6]. Since $L \cap H$ and $L \cap J$ have relatively prime orders, $L \cap H$ and $L \cap J$ are orthogonal with respect to $\psi$, so $\psi$ must be nondegenerate on both $L \cap H$ and $L \cap J$. We have $J_{\sigma} J_{\tau}=J_{\sigma \tau}$ [11], so we may write $S=\left(\Sigma_{\sigma \in(H \cap L)} J_{\sigma}\right) \otimes_{T}\left(\Sigma_{\sigma \in(J \cap L)} J_{\sigma}\right)=S_{H} \otimes_{T} S_{J}$. Clearly $S_{J} \subseteq S^{S_{H}}$, the commutator of $S_{H}$ in $S$; in fact, $S_{J}=S^{S_{H}}$ : for $S$ is an Azumaya $T$-algebra and $S_{H}$ and $S_{J}$ both have center $T$ by the nondegeneracy of $\psi$ on $L \cap H$ and $J \cap H$, and are contained in each other's commutators; [2, (3.3)] applies to yield $S_{J}=S^{S_{H}}, S_{H}=S^{S_{J}}$. Now for $x$ in $S, \sigma$ in $H \cap L, a$ in $J_{\sigma} \subseteq S_{H}$, we have $a x^{\sigma}=x a$. If $x$ is in $S^{H}, x^{\sigma}=x$ so $a x=x a$. So $S^{H} \subseteq$ $S^{S_{H}}=S_{J}$. Similarly, $S^{J} \subseteq S_{H}$. So elements of $S^{H}$ and of $S^{J}$ commute with each other.

Proof of (2.5). The difficulty in showing that if $S$ is in $\operatorname{Galz}_{\phi}(R, G)$ then $S^{J}$ is in $\operatorname{Galz}_{\phi_{H}}(R, H)$ lies in showing that the gradings of $S^{J}$ are in $H$. So we begin (2.5) with some lemmas on the gradings of $S$ in $\operatorname{Galz}_{\phi}(R, G)$.

(2.7) Lemma. Let $R$ be connected, $S$ in $\operatorname{Galz}_{\phi}(R, G)$. For each $\sigma$ in $K=\left\{\sigma \in G \mid S_{\sigma} \neq 0\right\}$ : 
(i) $S_{\sigma}=\left\{a \in S \mid a x^{\sigma}=\phi(a, x) x a\right\}$ is a rank one projective $\cdot S_{1}$-module,

(ii) $S_{\sigma} S_{\tau}=S_{\sigma \tau}$.

Proof of (2.7). Since $S$ is in $\operatorname{Galz}_{\phi}(R, G), S$ is a graded Azumaya $S_{1}$-algebra, by (1.1).

Let ${ }^{e} S=S^{\#} \otimes_{S_{1}} S$ [CGO, (1.3)] and view $S$ as an $S$-S bimodule, $S(1, \sigma)$, by the usual left $S$-module action of $S$ on $S$ and a right action by $s \cdot x=s x^{\sigma}$. Then the Morita equivalence [CGO, 2.8] between $S_{1}$-modules and right ${ }^{e} S$-modules shows that $J_{\sigma}=\left\{a \in S \mid a x^{\sigma}=\phi(a x) x a\right\}={ }^{S} S(1, \sigma)$ is a rank one projective $S_{1}$-module. Now $J_{\sigma}=S_{\sigma}$. For clearly $S_{\sigma} \subseteq J_{\sigma}$; on the other hand, if $a$ is in $S_{\rho}, a x^{\rho}=\phi(a, x) x a$ for all $x \in S$, so $a \in J_{\sigma} \cap S_{\rho}$ iff $x^{\rho}$ $=x^{\sigma}$ for all $x \in S$. But $S$ is a Galois extension of $R$ with group $G$, so if $x^{\rho}=x^{\sigma}$ for all $x$ in $S, \rho=\sigma$. Thus $J_{\sigma}=S_{\sigma}$. That $S_{\sigma} S_{\tau}=J_{\sigma} J_{\tau}=J_{\sigma \tau}=$ $S_{\sigma \tau}$ follows by tracing through the maps in the isomorphism of $J_{\sigma} \otimes J_{\tau}$ with $J_{\sigma \tau}$ in [15, Lemma 5] (as was observed in [7, Lemma 1]).

(2.8) Lemma. Assume $R$ is connected. If $K=\left\{\sigma \in G \mid S_{\sigma} \neq 0\right\}$ then $K$ is a group and $S$ is a Galois extension of $S_{1}$ with group $K$.

Proof of (2.8). From (2.7) (ii) it is clear that $K$ is a submonoid of $G$, so since $G$ is finite, $K$ is a group. Now note that $S_{1} \subseteq S^{K}$. For if $\sigma$ is in $K, a$ is in $S_{\sigma}, x$ is in $S_{1}$, then $a x^{a}=\phi(a, x) x a$. But $S_{1}$ is in the center of $S$ (use the same formula with $x$ arbitrary and $a$ in $S_{1}$ ), so $a x=x a$, so $a\left(x^{a}-x\right)=0$. Choose $a_{i}$ in $S_{\sigma}, b_{i}$ in $S_{\sigma^{-1}}$ with $\Sigma b_{i} a_{i}=1$; then $0=$ $\Sigma b_{i} a_{i}\left(x^{a}-x\right)=x^{a}-x$. Thus $S_{1} \subseteq S^{K}$. Now, since $S$ is a Galois extension of $S^{K}$ with group $K, S^{K}$ is a direct summand of $S, S$ is a projective $S^{K}$-module [4] and $\operatorname{rank}_{S_{1}} S=[K: 1]=\operatorname{rank}_{S}(S)$. Since $S^{K}$ is $S_{1}$-projective, $S_{1}$ is an $S_{1}$-direct summand of $S^{K}$. Thus $S^{K}=S_{1}$.

Proof of (2.5). We check the conditions of (1.1) for $T=S^{J}$. The right action of $H$ on $T$ is clear. We must show that the set of gradings of $T$ is contained in $H$.

We first observe that $T$ is a projective $T_{1}$-module. For this it suffices to assume $R$ is local. Now $T$ is a Galois extension of $R$ with group $H=G$ restricted to $T$, and $T_{1}$ is the fixed ring of $K$ restricted to $T$ since $T_{1}=$ $T \cap S_{1}$, by (2.8). So $T$ is a Galois extension of $T_{1}$ with group $K \cap H$; hence $T$ is $T_{1}$-projective, and $\operatorname{rank}_{T_{1}}(T)$ divides the order of $H$ at each prime ideal of $T_{1}$.

To show that the set of gradings of $T$ is contained in $H$ it suffices to assume $T_{1}$ is local. We have $T=\Sigma_{\sigma \operatorname{in} L} \bigoplus T_{\sigma}$, where $L=\left\{\sigma \in G \mid T_{\sigma} \neq 0\right\}$, so the $T_{\sigma}$, being $T_{1}$-submodules of $T$, are $T_{1}$-projective. The multiplication map $T \otimes_{T_{1}} S_{1} \rightarrow T S_{1}$ is an isomorphism since both are Galois extensions of 
$S_{1}$ with group $H \cap K \cong K /(J \cap K)$ [4, (3.4)]. This map sends $T_{\sigma} \otimes_{T_{1}} S_{1}$ one-one into $S_{\sigma}$. Since $S_{\sigma}$ is a rank one projective $S_{1}$-module, $T_{\sigma}$ must be a rank one projective $T_{1}$-module. We show $T_{\sigma} \cdot T_{\tau} \neq 0$, which will suffice to show that $L$ is a group whose order $=\operatorname{rank}_{T_{1}}(T)$ divides the order of $H$; hence $L \subseteq H$.

To show $T_{\sigma} \cdot T_{\tau} \neq 0$ it suffices to show that $T_{\sigma} S_{1} \cdot T_{\tau} S_{1} \neq 0$, since $S_{1}$ is in the center of $S$. But $T_{\sigma} S_{1}$ and $T_{\tau} S_{1}$ are rank one projective $S_{1}$ submodules of the rank one projectives $S_{\sigma}$ and $S_{\tau}$. Since $S_{1}$ is semilocal, we may pick free bases $S_{\sigma}=S_{1} x, S_{\tau}=S_{1} y, T_{\sigma} S_{1}=S_{1} c x, T_{\tau} S_{1}=S_{1} d y, x, y \in S$, $c, d$ in $S_{1}$; then $S_{\sigma} S_{\tau}=S_{\sigma \tau}=S_{1} x y$. Since $x, y, c x, d y$ and $x y$ are bases for free $S_{1}$-modules of rank 1 , none can be divisors of zero in $S_{1}$. Thus $T_{\sigma} S$ $T_{\tau} S_{1}=S_{1} c d x y \neq 0$, for as is easily seen, $c d x y$ cannot be a zero divisor. Thus $L \subseteq H$.

To show that $T$ is a left $H$-module observe that since $T$ is graded by $H$, for all $a$ in $T, \sigma$ in $J, a^{\sigma}={ }^{\sigma} a$, by (1.1), (4). Thus $S^{J}=T \subseteq{ }^{J} S$. The leftright symmetry of the argument thus far gives the opposite inclusion. Thus $T=$ ${ }^{J} S$ and has a natural left $H$-action induced from the left action of $G$.

It is clear then that (2r) and (2l) of (1.1) hold for $T$, and that (3r), (3l) and (4) hold by inheritance from $S$. That proves (2.5).

Proof of (2.6). With $G=H \times J$ as above, let $S, T$ be in $\operatorname{Galz}_{\phi}(R, G)$. Then $S=S^{J} \otimes S^{H}, T=T^{J} \otimes T^{H}$ with $S^{J}, T^{J}$ in $\mathrm{Galz}_{\phi}(R, H), S^{H}, T^{H}$ in $\mathrm{Galz}_{\phi_{J}}(R, J)$, by (2.4) and (2.5). We have $S \cdot T=S^{J} \cdot T^{\mathcal{S}} \otimes S^{H} \cdot T^{H}$. For $H$ and $J$ are mutually orthogonal with respect to $\phi$, so the usual "switch" map

$$
S \otimes T=\left(S^{J} \otimes S^{H}\right) \otimes\left(T^{J} \otimes T^{H}\right) \cong\left(S^{J} \otimes T^{J}\right) \otimes\left(S^{H} \otimes T^{H}\right)
$$

is an $R$-algebra isomorphism. Then if $D G$, etc., is the kernel of the multiplication map from $G \times G$ to $G$, acting on $S \otimes T$ by $\left(\sigma^{-1}, \sigma\right)(s \otimes t)=s^{\sigma} \otimes{ }^{\sigma}$ (cf. proof of (1.2)) it is easy to see that if $(S \otimes T)^{D G}=S \cdot T$ is the fixed ring under the action of $D G$, then $(S \otimes T)^{D G} \cong\left(S^{J} \otimes T^{J}\right)^{D H} \otimes\left(S^{H} \otimes T^{H}\right)^{D J}$. This completes the proof of (2.6) and hence of (2.1).

3. Matrix computations. In view of Corollary (2.2) in order to describe $\mathrm{Galz}_{\phi}(R, G)$, it is enough to do it for $p$-groups. This section will be devoted to a description of $\mathrm{Galz}_{\phi}(R, G)$ for $G$ a $p$-group which is a finite product of cyclic groups of equal exponent, i.e., groups which are free modules of finite rank over $Z / p^{e} Z$. Before restricting ourselves to such $G$ we first obtain a general remark about elements of $\operatorname{Galz}(R, G)$ under the following assumption, which will remain in force throughout this section:

(3.1) Assume $G$ is a finite abelian group of order $n$ and exponent $m$, 
and $R$ is a connected commutative ring containing $1 / n$ and a primitive $m$ th root of unity.

Under the assumption (3.1) $G^{*}=\operatorname{Hom}(G, U(R)) \cong G$, and we may decompose any abelian Galois extension, just as in classical Kummer Theory of fields, into a direct sum of $R$-submodules, indexed by $G^{*}$, on each of which each element of $G$ acts as multiplication by a root of unity [6].

Let $S$ be in $\operatorname{Galz}(R, G)$. Then $S$ is graded in three ways: by $G$, and by $G^{*}=\operatorname{Hom}(G, U(R))$ in two different ways, arising from the left and right actions of $G$ on $S$, respectively, as follows: Since $S$ is, with respect to each of the $G$-actions, a Galois extension of $R$ with group $G, S=\Sigma_{x} S_{x}=\Sigma_{x} x^{S}$ with respect to the $G^{*}$-gradings, where

$$
S_{\chi}=\left\{s \text { in } S \mid s^{\sigma}=\chi(\sigma) s\right\}, \quad \chi^{S}=\left\{s \text { in }\left.S\right|^{\sigma} s=\chi(\sigma) s \text { for all } \sigma \text { in } G\right\} .
$$

(3.2) Lemma. Each $S_{x}$ is a ${ }_{\psi} S$ and each is homogeneous with respect to $G$.

Proof. Since the left action by $G$, the right action by $G$, and the grading by $G$ all commute with each other, we may view $S$ as an $R G \otimes R G \otimes$ $R G^{*}$-module, hence as an $R G^{*} \otimes R G^{*} \otimes R G$-comodule, i.e., as graded by $G^{*} \times$ $G^{*} \times G$. Thus

$$
S=\sum_{(\psi, \chi, \sigma)} S_{(\psi, \chi, \sigma)}
$$

$\left(S_{(\psi, \chi, \sigma)}=\right.$ elements homogeneous of degree $(\psi, \chi, \sigma)$ on $\left.G^{*} \times G^{*} \times G\right)$ and, in particular, each $S_{\chi}=\Sigma_{(\psi, \sigma)} S_{(\psi, x, \sigma)}$; each ${ }_{\psi} S=\Sigma_{(\chi, \sigma)} S_{(\psi, x, \sigma)}$. But each $S_{\chi}$, respectively ${ }_{\psi} S$, is a rank one projective $R$-module, so that (since $R$ is connected) there can be only one summand. Thus $S_{x}=S_{(\psi, x, \sigma)}$ for some $\psi, \sigma$; also ${ }_{\psi} S=S_{(\psi, \chi, \sigma)}$ for some $\chi, \sigma$. Thus if $S_{\chi}=S_{(\psi, \chi, \sigma)}, S_{\chi}={ }_{\psi} S \subseteq$ $S_{\sigma}$.

We now assume that $G$ is a $p$-group. We wish to compute $\operatorname{Galz}_{\phi}(R, G)$. This is relatively complicated in general, but becomes manageable in case

(3.3) $G$ is a product of cyclic groups all of the same order $m=p^{e}$.

For in that case we can map $\operatorname{Galz}(R, G)$ into a group of orthogonal matrices over $Z / p^{e} Z$. We now describe how this is done.

Let $G=\Pi_{i=1}^{r} Z_{i}$ with $Z_{i}=\left\langle\sigma_{i}\right\rangle$, cyclic of order $p^{e}$, and $G^{*}=\Pi_{i=1}^{r} Z_{i}^{*}$ with $Z_{i}^{*}=\left\langle\chi_{i}\right\rangle$, where, if $\zeta$ is a fixed $m$ th root of unity, $\chi_{i}\left(\sigma_{i}\right)=\zeta$. Let $S$ be in $\operatorname{Galz}_{\phi}(R, G)$.

From [6] and (3.1) we can write $S=\Sigma_{\chi \in G} S_{x}$ where $G$ acts on $s$ in $s_{\mathrm{\chi}}$ by $s^{\tau}=\chi(\tau)$ s. By (3.2), $S_{\chi}=S_{(\psi, \chi, \sigma)}$, that is, $\tau_{s}=\psi(\tau) s$ and $s$ is 
homogeneous of grade $\sigma$. By [6] all of these gradings respect the multiplication in $S$; hence

$$
S_{\left(\psi_{1}, \chi_{1}, \sigma_{1}\right)} \cdot S_{\left(\psi_{2}, \chi_{2}, \sigma_{2}\right)}=S_{\left(\psi_{1} \psi_{2}, \chi_{1} x_{2}, \sigma_{1} \sigma_{2}\right)} \cdot
$$

We will associate to $S$ some $r \times r$ matrices with entries in $Z / m Z$.

Notation. Let $\chi=\left(\chi_{1}, \cdots, \chi_{r}\right), \sigma=\left(\sigma_{1}, \cdots, \sigma_{r}\right)$ and, if $\beta$ is a vector $\left(\beta_{1}, \cdots, \beta_{r}\right)$ in $(Z / m Z)^{r}$, write $\chi^{\beta}=\chi_{1}^{\beta_{1}} \chi_{2}^{\beta_{2}} \cdots \chi_{r}^{\beta_{r}}$, and similarly for $\sigma^{\beta}$.

For each $i, S_{\chi_{i}}=S_{\left(x^{p i, x^{e}} e_{, \sigma} \alpha_{i}\right)}$ where $p_{i}, \epsilon_{i}, \alpha_{i}$ are (row) vectors in $(Z / m Z)^{r}, \epsilon_{i}=(0, \cdots, 1, \cdots 0)(1$ in $i$ th entry $)$. Now $S$ is generated as an algebra by the $S_{x_{i}}$, so the left $G$-action on $S$ is described by the $r \times r$ matrix

$$
P=\left(\begin{array}{c}
p_{1} \\
\vdots \\
p_{r}
\end{array}\right)
$$

of left $G^{*}$-gradings of the $S_{\chi_{i}} i=1, \cdots, r$, and similarly the $G$-grading on $S$ is described by the $r \times r$ matrix

$$
A=\left(\begin{array}{c}
\alpha_{1} \\
\vdots \\
\alpha_{r}
\end{array}\right)
$$

of $G$-gradings of the $S_{\chi_{i}}$. The corresponding matrix of right $G^{*}$-gradings is

$$
\left(\begin{array}{c}
\epsilon_{1} \\
\vdots \\
\epsilon_{r}
\end{array}\right)=I \text {. }
$$

Let $u_{i}$ be a typical element of $s_{x_{i}}$ for $i=1, \cdots, r$. From [6] (cf. proof of (2.4) above) we know that $u_{i} u_{j}=\delta\left(\chi_{i}, \chi_{j}\right) u_{j} u_{i}$ where $\delta: G^{*} \times G^{*}$ $\rightarrow U(R)$ is a skew bilinear map; $\delta$ thus describes the way elements of $S$ (fail to) commute with each other. By bilinearity, $\delta\left(\chi_{i}, x_{j}\right)=\xi^{d_{i j}}$ and $D=$ $\left(d_{i j}\right)$ is clearly an $r \times r$ skew-symmetric matrix with entries in $Z / m Z$.

From bilinearity of $\phi$ we have $\phi\left(\sigma_{i}, \sigma_{j}\right)=\xi^{\phi_{i j}}$ where $\phi=\left(\phi_{i j}\right)$ is an $r \times r$ matrix in $Z / m Z$. Set $M=\phi+\phi^{\prime}$ where ' means transpose.

The structure of $S$ as an element of $\operatorname{Galz}_{\phi}(R, G)$ defines some relations among $P, A, D, \phi, M$.

The relations:

(1) $u_{i} u_{j}^{u_{i}}=\phi\left(u_{i}, u_{j}\right) u_{j} u_{i}$,

(2) $u_{i} u_{j}=\delta\left(x_{i}, x_{j}\right) u_{j} u_{i}$ with $\delta$ skew bilinear,

(3) $u_{i}^{\sigma}=\phi\left(\sigma, u_{i}\right) \phi\left(u_{i}, \sigma\right)^{\sigma} u_{i}$,

(4) $S$ is a left Galois extension with group $G$, for all $i, j, \sigma$ are necessary in order that $S$ be in $\operatorname{Galz}_{\phi}(R, G)$. 
Condition (4) is equivalent to the nonsingularity of $P$.

Conditions (1) and (2) combine to yield:

$$
\begin{aligned}
& u_{i} u_{j}^{u_{i}}=\phi\left(u_{i}, u_{j}\right) \delta\left(\chi_{j}, \chi_{i}\right) u_{i} u_{j}, \quad \text { i.e., } \\
& \left\langle\chi^{\epsilon}, \sigma^{\alpha}{ }^{i}\right\rangle=\phi\left(\sigma^{\alpha}, \sigma^{\alpha}\right) \delta\left(\chi_{j}, \chi_{i}\right) \text {, i.e., } \\
& \zeta^{\alpha_{i} \epsilon_{j}^{\prime}}=\xi^{\alpha_{i} \phi \alpha_{j}^{\prime} d_{j i}}, \text { i.e., } \\
& d_{i j}+\alpha_{i} \epsilon_{j}^{\prime}=\alpha_{i} \phi \alpha_{j}^{\prime} \text {, i.e., } \\
& D+A I=A \phi A^{\prime} \text {. }
\end{aligned}
$$

Condition (3) becomes

$$
\begin{aligned}
\left\langle\chi^{\epsilon_{i}}, \sigma_{j}\right\rangle & =\phi\left(\sigma_{j}, \sigma^{\alpha_{i}}\right) \phi\left(\sigma^{\alpha_{i}}, \sigma_{j}\right)\left\langle\chi^{\rho_{i}}, \sigma_{j}\right\rangle \text {, i.e., } \\
\epsilon_{i} \epsilon_{j}^{\prime} & =\epsilon_{j} \phi \alpha_{i}^{\prime}+\alpha_{i} \phi \epsilon_{j}^{\prime}+\rho_{i} \epsilon_{j}^{\prime}, \quad \text { i.e., } \\
I & =A \phi^{\prime}+A \phi+P .
\end{aligned}
$$

Summing up, conditions (1)-(4) yield:

$$
\begin{aligned}
& D=A \phi A^{\prime}-A \text { is skew, } \\
& P=I-A M \text { is nonsingular. }
\end{aligned}
$$

(3.4) THEOREM. With $\dot{R}, G$ as in (3.1), (3.3), $\operatorname{Galz}(R, G)$ is described by the following two exact sequences:

$$
\begin{gathered}
1 \rightarrow \operatorname{Galz}_{\phi}^{0}(R, G) \rightarrow \operatorname{Galz}_{\phi}(R, G) \stackrel{\beta}{\longrightarrow} O_{Z / m Z}^{r}(M), \\
1 \rightarrow \operatorname{Comm}(R, G) \rightarrow \operatorname{Galz}_{\phi}^{0}(R, G) \stackrel{\nu}{\longrightarrow} \operatorname{Skew}_{Z / m Z}^{r}(M),
\end{gathered}
$$

where $O_{Z / m Z}^{r}(M)=\left\{P \in G L_{r}(Z / m Z) \mid P^{\prime} M P=M\right\}$ and $\beta$, defined by $\beta(S)=P$, is an antihomomorphism, $\operatorname{Skew}_{Z / m Z}^{r}(M)=\left\{A \in M_{r}(Z / m Z) \mid A M=0\right.$ and $A^{\prime}=$ $-A$ \} and $\nu$, defined by $\nu(S)=A$, is a homomorphism.

If $M=0, \beta=1$.

If $p \neq 2, \nu$ is onto; if $M$ is nonsingular, $\nu=0$ and $\beta$ is onto.

If $p=2, \operatorname{Im} \nu=\left\{A \in \operatorname{Skew}^{r}(M) \mid\left(A \phi A^{\prime}-A\right)_{i i}=0\right.$ for all $\left.i=1 \cdots r\right\}$; if $M$ is nonsingular, $\nu=0$ and $\operatorname{Im} \beta=\left\{P \in O^{r}(M) \mid\left[(I-P) M^{-1} \phi M^{-1}(I-P)\right.\right.$ $\left.-\left(I-P^{\prime}\right) M^{-1}\right]_{i i}=0$ for $\left.i=1 \cdots r\right\}_{\text {. }}$

We remark that $\operatorname{Galz}_{\phi}^{0}(R, G)$ is a subgroup of $\operatorname{Galz}_{\phi}(R, G)$ whose product $S \cdot T=\left(S \otimes_{R} T\right)^{D}$ is the usual product of Galois extensions. This follows 
by $[C G O,(3.6)]$ and the fact that since $P=I$ the left and right $G$-actions on $S$ in $\operatorname{Galz}_{\phi}^{0}(R, G)$ coincide.

Proof. Since $A \phi A^{\prime}-A$ is skew, $A M A^{\prime}=A+A^{\prime}$. Thus $P A^{\prime}=A^{\prime}-$ $A M A^{\prime}=-A, P^{-1} A=-A^{\prime}$, so

$$
\begin{aligned}
P^{\prime} M P & =\left(I-M A^{\prime}\right) M P \quad \text { (since } M \text { is symmetric) } \\
& =M P-M A^{\prime} M P=M P+M P^{-1} A M P=M P+M P^{-1}(I-P) P \\
& =M P+M P^{-1} P-M P^{-1} P P=M .
\end{aligned}
$$

Thus $\beta$ is well defined. If $P=I, A M=0$ and $A+A^{\prime}=A M A^{\prime}=0$. Thus $\nu$ is well defined.

We show that the map $\beta$ is an antihomomorphism.

Let $S, T$ be in $\operatorname{Galz}(R, G)$ with left grading matrices $P, Q$, respectively. Then $S T=\Sigma S_{\psi} \otimes_{\psi} T$, where $\psi$ runs through $G^{*}$. Since the right grading on $S T$ is induced from that on $T$, and the left grading is induced from that on $S$, we can compute the matrix of $S T$ by noticing that $T_{x_{i}}={ }_{x^{q} i} T$, and $S_{x_{i}}=$ ${ }_{x}^{p} S$, so that $(S T)_{x_{i}}=S_{x_{i}} \otimes T_{x_{i}}$, and the left grade of

$$
S_{\chi^{q_{i}}}=S_{\chi_{1}}^{q_{i 1}} S_{\chi_{2}}^{q_{i 2}} \cdots s_{\chi_{r}}^{q_{i r}} \text { is } \chi^{p_{1} q_{i 1}+p_{2} q_{i 2}+\cdots+p_{r} q_{i r}}=\chi^{q_{i} P} .
$$

So the left grading matrix of $S T$ is $Q P$.

If $S, T$ have left grading matrices $P=Q=I$, then the $G$-grading on $S T$ is that of $(S \cdot T)_{x_{i}}=S_{x_{i}} \otimes_{x_{i}} T=S_{x_{i}} \otimes T_{x_{i}}$, namely, $\sigma^{\alpha_{i}+\beta_{i}}$. So $\nu$ is a homomorphism.

If $\nu(S)=0$ then $S$ is trivially graded and $D=0$, so $S$ is commutative. On the other hand, it is easy to check that any trivially graded commutative Galois extension is in $\operatorname{Galz}^{0}(R, G)$. Thus the two sequences are exact. Since $P=I-A M$, if $M=0, \beta=1$. All that is left to prove are the ontoness statements for $\nu$ and $\beta$.

We begin showing ontoness of $\beta$, assuming $M$ is invertible, by picking $P$ in $O(M)$ and defining $A$ by $A=(I-P) M^{-1}$. Then $P^{\prime} M P=M, P M^{-1} P^{\prime}=$ $M^{-1}$, that is, $M^{-1}=(I-A M) M^{-1}\left(I-A M^{\prime}\right)=M^{-1}-A-A^{\prime}+A M A^{\prime}$, so $A \phi A^{\prime}-A=D$ is skew. If $p=2$ we assume $D$ has zero diagonal. Thus associated to $P$ are matrices $A, D$. We begin showing ontoness of $\nu$ by letting $A$ be such that $A M=0$ and $A^{\prime}=-A$. Then associated to $A$ are the skew matrix $D=A \phi A^{\prime}-A$ and the nonsingular matrix $P=I=I-A M$. If $p=2$ we assume $D$ has zero diagonal.

Given $P, A, D$ we define an algebra $S$ in $\operatorname{Galz}_{\phi}(R, G)$ whose structure is described by these matrices as follows.

Let $S=\Sigma_{\nu} R u_{1}^{\nu_{1}} u_{2}^{\nu_{2}} \cdots u_{r}^{\nu_{r}}$, where $u_{i}^{m}=1$. Fixing as above a primitive 
$m$ th root of unity $\zeta$, define $u_{i} u_{j}=\zeta^{d}{ }_{i j} u_{j} u_{i}$, extending by linearity. This defines the multiplication on $S$. Since $d_{i i}=0$ it is well defined. Define gradings on $S$ by giving $u_{i}$ the left $G^{*}$-grading $\chi^{p_{i}}$, the right $G^{*}$-grading $\chi^{\epsilon_{i}}$, and $G$-grading $\sigma^{\alpha}$. It is then clear that $S$ is a $G$-graded $R$-algebra and a Galois extension on the right with group $G$. Since $P$ is invertible, one can find elements $v_{i}$ in $S$ so that $v_{i}$ has left grading $\chi_{i}$ and the $v_{i}$ generate $S$ in the same way as the $u_{i}$. So $S$ is a Galois extension on the left with group $G$. The other relations (1)-(4) of (1.1) all follow from the relationships among the matrices $P, A, \phi, D$. This shows the ontoness of $\nu$, and if $M$ is invertible, the ontoness of $\beta$, completing the proof of the theorem.

(3.5) Remark. When $R, G$ satisfy (3.1) the description of $\operatorname{Im}(\pi)$ for $G$ a cyclic $p$-group given in [CGO, Theorem 4.1] follows easily from the techniques of Theorem (3.4) and the result, to be proved in $\S 4$, that, for $G$ a cyclic $p$-group, $\operatorname{Im}(\pi)=\operatorname{Galz}(R, G)$. For suppose $G$ is a cyclic $p$-group with generator $\sigma$, with the order of $G=p^{e}=m$. Choose $\zeta$, a primitive $p^{e}$ th root of unity, so that $\phi(\sigma, \sigma)=\zeta^{p^{i}}$ for some $i \geqslant 0$. Note that in applying (3.4) all matrices are $1 \times 1$.

For $p$ odd, (3.4) reads:

$$
1 \rightarrow \operatorname{Comm}(R, G) \rightarrow \operatorname{Galz}_{\phi}(R, G) \stackrel{\beta}{\longrightarrow} O_{Z / p}^{1} e_{Z}^{\left(\left(2 p^{l}\right)\right)} \rightarrow 0
$$

since $\operatorname{Skew}_{Z / p^{e} Z}^{1}\left(\left(2 p^{i}\right)\right)=0$. Now $O_{Z / m Z}^{1}\left(\left(2 p^{i}\right)\right)=Z / 2 Z$ unless $\phi=1$. If $i=0, \beta$ is onto by (3.4) and we recover [CGO, 4.1]. If $i>0$ then since $D=$ $A \phi A^{\prime}-A$ is skew, $D=0$, so $A\left(\phi A^{\prime}-I\right)=0$, so $A=0, P=I$ since $I-$ $\phi A^{\prime}$ is a unit. So if $i>0, \beta=1$ and we recover [CGO, 4.1].

For $p=2, G$ of order $p^{e}, e>1$, set $\phi(\sigma, \sigma)=\zeta^{2^{i}}$. Now $\{A \in$ Skew $\left.^{1}(M) \mid A \phi A^{\prime}-A=0\right\}=\{0\}$, as is easily checked, so we simply have to compute $\beta$. We know $0=D=A(\phi A-I)$ since cyclic Galois extensions are commutative. If $i \geqslant 1, \phi A-I$ is a unit and $A=0, \beta=1$. If $i=0, A(A-I)=0$ so $A=I$ or $A=0$. If $A=I$, since $M=(2), P=-I$, otherwise $P=I$. So if $i \geqslant 1, \operatorname{Galz}_{\phi}(R, G)=\operatorname{Comm}(R, G)$; if $i=0$ we get that $\operatorname{Im}(\beta)=Z / 2 Z$ and

$$
1 \rightarrow \operatorname{Comm}(R, G) \rightarrow \operatorname{Galz}(R, G) \rightarrow Z / 2 Z \rightarrow 1
$$

is exact, recovering [CGO, 4.1] in this case.

For $p=2, G=Z / 2 Z, \phi(\sigma, \sigma)=(-1)^{2^{i}}(i=0$ or 1$)$, and $M=0$, so $\beta=1$ and the sequence (3.4.2) describes $\operatorname{Galz}_{\phi}(R, G)$. Then it is easily checked that $\left\{A \in \operatorname{Skew}^{1}(M) \mid A \phi A^{\prime}-A=0\right\}$ is equal to $\{0\}$ if $i=1$, i.e., $\phi$ is trivial, but equals $\{0,1\}$ if $i=0$, i.e., $\phi$ is nontrivial, thereby recovering [CGO, 4.1] in this case also.

4. Ontoness of $\pi$. In this section we prove, under appropriate conditions 
on $R$, that $\pi$ is onto. This result, together with those of $\S 3$, yields a description of $B_{\phi}(R, G)$ for any $G=\Pi Z_{p} e$

Throughout this section, the assumptions (3.1) hold, i.e., $G$ is a group of order $n$ and exponent $m$, and $R$ is a connected commutative ring containing $1 / n$ and a primitive $m$ th root of unity.

We begin with some results on the structure of elements of $\operatorname{Galz}_{\phi}(R, G)$.

(4.1) Lemma. With $R, G$ as above, let $S$ be in $\operatorname{Galz}_{\phi}(R, G)$, and let $K=\left\{\sigma \in G \mid S_{\sigma} \neq 0\right\}$ be the group (2.8) of gradings of $S: S=\Sigma_{\sigma \in K} S_{\sigma}$. Let $Z$ be the ungraded center of $S$ and let $H=\left\{\sigma \in K \mid Z_{\sigma} \neq 0\right\}$. Then $H$ is a group and $Z_{\sigma}=S_{\sigma}$ for all $\sigma$ in $H$.

Proof. It suffices to verify both assertions when $R$ is local. In that case, $S$, being a Galois extension of $R$ with group $G$, is a twisted group ring $S=$ $\Sigma_{\chi \in G *} R u_{\chi}$ with factor set in $U(R)$. The noncommutativity of $S$ is expressed by $u_{\chi} u_{\psi}=D(\chi, \psi) u_{\psi} u_{\chi}$, where $D: G^{*} \times G^{*} \rightarrow U(R)$ is skew and bilinear [6].

Now for any $\chi, \psi$ in $G^{*}, u_{\chi} u_{\psi}^{u} \chi=\phi\left(u_{\chi}, u_{\psi}\right) D(\psi, \chi) u_{\chi} u_{\psi}$; if $u_{\chi}$ has grading $\sigma_{x}$ this becomes $\left\langle\psi, \sigma_{\chi}\right\rangle=\phi\left(\sigma_{x}, \sigma_{\psi}\right) D(\psi, \chi)$, that is, as a function of $\chi, D$ only depends on the grading of .u. Thus, $H=\{\sigma \in K \mid\langle\psi, \sigma\rangle=$ $\phi\left(\sigma, \sigma_{\psi}\right)$ for all $\psi$ in $\left.G^{*}\right\}$, a group, and if $\sigma \in H$, every $u_{x}$ in $S_{\sigma}$, hence all of $S_{\sigma}$ (in view of (3.2)) is in $Z$.

(4.2) LEMmA. If $S \in \mathrm{Galz}_{\phi}(R, G), R, G$ as above, if $Z$ is the ungraded center of $S$ and

$$
C=\left\{x \in S \mid x^{\sigma}=\phi(\sigma, x) x \text { for all } \sigma \text { in } G\right\},
$$

then $Z=C \cdot S_{1} \cong C \otimes_{R} S_{1}$.

Proof. We know that $S$ is a Galois extension of $R$ with group $G$ acting on the right, the image of $\sigma$ in $G$ on $s \in S$ being written $s^{\sigma}$. Define a new action of $G$ on $S$ by $\sigma(s)=\phi\left(\sigma^{-1}, s\right) s^{\sigma}$. Denote $G$ when acting in that way on $S$ by $G_{t}$. Notice that $C=\left\{s \in S \mid \sigma(s)=s \forall \sigma \in G_{t}\right\}$ by definition. Also, $\{\sigma \mid \sigma(s)=s$ for all $s \in S\}=H$. For if $\sigma \in G$ and $\sigma(s)=s$ for all $s$ in $S$, then in particular $\sigma(s)=s$ for all $s$ in $S_{1}$, but on $S_{1}, \sigma(s)=$ $s^{\sigma}$, so $\sigma \in K$ by (2.8). Now for $\sigma$ in $K, x$ in $S_{\sigma}, x s^{\sigma}=\phi(\sigma, s) s x$, so $x \sigma(s)=s x$. Thus $\sigma(s)=s$ for all $s$ in $S$ iff for all $x$ in $S_{\sigma}, s$ in $S, x s=$ $s x$, iff $\sigma \in H$, since $H$ is the group of gradings of the center of $S$.

Now we observe that, for each $\sigma$ in $(G / H)_{t}, \sigma(s)=s^{\tau}$ for some $\tau$ in $G$. For $\sigma(s)=\phi\left(\sigma^{-1}, s\right) s^{\sigma}$. We know from $\S 3$ that $S=\Sigma_{\chi \in G^{*}} S_{\chi}$ such that if $u_{\chi} \in S_{\chi}, \sigma \in G$, then $u_{\chi}^{\sigma}=\langle\chi, \sigma\rangle u_{\chi}$. Now $\sigma\left(u_{\chi}\right)=\phi\left(\sigma^{-1}, \operatorname{gr}\left(u_{\chi}\right)\right)\langle\chi, \sigma\rangle u_{\chi}$ (where $\operatorname{gr}\left(u_{\chi}\right)=$ the grade of $u_{\chi}$ ). The map $\sigma: G^{*} \rightarrow U(R)$ given by $\sigma(\chi)=\phi\left(\sigma^{-1}, \operatorname{gr}\left(u_{\chi}\right)\right)\langle\chi, \sigma\rangle$ is a homomorphism. Thus $\sigma \in \operatorname{Hom}\left(G^{*}, U(R)\right)=$ 
$G$, that is, there exists $\tau_{\sigma} \in G$ such that $\left\langle\chi, \tau_{\sigma}\right\rangle=\phi\left(\sigma^{-1}, \operatorname{gr}\left(u_{\chi}\right)\right)\langle\chi, \sigma\rangle$ for all $\chi \in G^{*}$. This means that $\sigma(s)=s^{\tau_{\sigma}}$ for some $\tau_{\sigma}$ in $G$. Clearly this map $\sigma \mapsto \tau_{\sigma}$ is a homomorphism. Let $L$ be the image in $G$ of the map $\sigma \mapsto \tau_{\sigma}$. Then $L=(G / H)_{t}$, so that, with respect to the usual right action of $G$ on $S$, $C$ is the fixed ring of some subgroup $L$. Thus, by $[4,(2.2)], S$ is a Galois extension of $C$ with group $L=(G / H)_{t}$, and $C$ is a Galois extension of $R$ with group $G / L$, and $([4,(4.1)]) \operatorname{rank}_{R} C=[G: L]=[H: 1]$.

Now in particular, $C$ is $K / H^{\perp} \cap K=\operatorname{Gal}\left(Z / S_{1}\right)$-strong $[4,(2.1)]$ since $C$ is $G$-strong [4, (2.2)] and $H^{\perp} \cap K$ leaves $C$ fixed. (Here $K$ is as in (4.1) or (2.8) and $H^{\perp}=\{\sigma \in G \mid \phi(\sigma, \tau)=1$ for all $\tau$ in $H\}$.) So the restriction of $K / H^{\perp} \cap K$ to $C$ acts as a subgroup of $\mathrm{Gal}(C / R)$ with fixed ring $C \cap S_{1}$. But $C \cap S_{1}$ is easily seen to be $R$. So $\operatorname{Gal}(C / R)$ is the restriction of $K / H^{\perp}$ $\cap K$ to $C:\left(K / H^{\perp} \cap K\right) / \bar{J} \cong \mathrm{Gal}(C / R)$, the isomorphism being by restriction, where $\bar{J} \subseteq K / H^{\perp} \cap K$ leaves $C$ fixed. In fact, since $\operatorname{rank}_{R} C=\operatorname{rank}_{S_{1}} Z=$ $[H: 1], \overline{\bar{J}}=\{1\}$. Now $\left(K / H^{\perp} \cap K\right)$ acts as a group of automorphisms of $C S_{1}$ with fixed ring $S_{1}$, and the Galois elements $([4,(1.3 \mathrm{~b})]$, or see $\S 1)$ for $C / R$ are at the same time Galois elements for $S_{1} C / S_{1}$. So $C S_{1}$ is a Galois extension of $S_{1}$ with group $\left(K / H^{\perp} \cap K\right)$. Also $C \otimes_{R} S_{1}$ is a Galois extension of $S_{1}$ with group $K / H^{\perp} \cap K$. Thus two applications of [4, (3.4)] to the maps $C \otimes_{R}$ $S_{1} \rightarrow C S_{1} \subseteq Z$ show that $C \otimes_{R} S_{1} \cong Z$, proving the lemma.

We are ready for the main result of this section.

(4.3) Theorem. If $R, G$ are as above, $S$ is in $\operatorname{Galz}_{\phi}(R, G), H$ is the group of gradings of the center $Z$ of $S$, and either $H$ is a direct summand of $G$ or $\operatorname{Pic}(R)$ is $[H: 1]$-torsion free, then $S \in \operatorname{Im}(\pi)$.

Proof. If $C=\left\{x \in S \mid x^{\sigma}=\phi(\sigma, x) x \forall \sigma \in G\right\}$, we know by (4.2) that $C \otimes_{R} S_{1} \cong Z$, so for each $\sigma$ in $H, C_{\sigma} \otimes_{R} S_{1} \cong S_{\sigma}$. Thus $C_{\sigma}$ is a rank one projective $R$-module, and from the equality $S_{\sigma} S_{\tau}=S_{\sigma \tau}$, one obtains that $\left(C_{\sigma} S_{1}\right)\left(C_{\tau} S_{1}\right)=\left(C_{\sigma \tau} S_{1}\right)$, i.e., $C_{\sigma} C_{\tau} \otimes_{R} S_{1}=C_{\sigma \tau} \otimes_{R} S_{1}$; hence (since $R$ is an an $R$-direct summand of $\left.S_{1}\right) C_{\sigma} C_{\tau}=C_{\sigma \tau}$.

Thus if $H=\left\langle\tau_{1}\right\rangle \times \cdots \times\left\langle\tau_{r}\right\rangle$, a product of cyclic groups, and $H$ has order $n_{H}$, and if $\operatorname{Pic}(R)$ is $n_{H}$-torsion free, each $C_{\tau}$ is free and we may choose $u_{i}, i=1 \cdots r$, so that $u_{i} u_{j}=u_{j} u_{i}, C_{\tau_{i}}=R u_{i}$ and $u_{i}^{n_{i}}=a_{i} \in U(R)$ when $n_{i}=$ the order of $\tau_{i}$. That is, $C=R H_{f}$, a commutative twisted group ring with twisting $f$, a symmetric 2-cocycle representing a class in $H^{2}(H, U(R))$. (A map $f: G \times G \rightarrow U(R)$ is symmetric if $f(\sigma, \tau)=f(\tau, \sigma)$.)

We suppose, as we shall prove in (4.4), that there exists a symmetric 2-cocycle $g \in H^{2}(G, U(R))$ such that $\mathrm{res}_{G / H} g=f$, so that $C \cong R H_{f} \subseteq R G_{g}$ and $R G_{g}$ is commutative. Let $A=R G_{g} \otimes_{C} S$ with multiplication $s z_{\sigma}=\phi\left(\sigma^{-1}, s\right) z_{\sigma} s^{\sigma}$; for 
$z_{\sigma} \in C$, respectively (since $R G_{g}$ is commutative) for $s \in C$, this agrees with the usual multiplication of $C$ in $S$, respectively in $R G_{g}$. So the multiplication on $A$ is well defined. We must show that $A$ is separable over $R$, and for this it suffices to observe that $C$ is a Galois extension of $R$, hence $R$-separable, and to prove that $A$ is an Azumaya $C$-algebra. In fact, notice that if $\sigma(s)=\phi\left(\sigma^{-1}, s\right) s^{\sigma}$ is the $G_{t}$ action on $S$ defined in the proof of (4.2), then $s z_{\sigma}=z_{\sigma} \sigma(s)$. This suggests that $A$ may be viewed as a crossed product $\Delta\left(S,(G / H)_{t}\right)_{h}$ with some factor set $h: G / H \times G / H \rightarrow U(C)$; since $S$ is a Galois extension of $C$ with group $(G / H)_{t}$ (proof of (4.2)) it would follow that $A$ is an Azumaya $C$-algebra split by $S$.

To show $A=\Delta\left(S,(G / H)_{t}\right)_{h}$ we observe that we can find an $h \in$ $H^{2}(G / H, U(C))$ so that $R G_{g} \cong C(G / H)_{h}$. We do that by letting $v: G / H \rightarrow G$ be a set splitting of the canonical map: $G \rightarrow G / H$, with $v(\overline{1})=1$, and we define an $R$-module isomorphism $\psi: R G_{g} \rightarrow C(G / H)$ induced by linearity and $\psi(\sigma)=\left(v(\bar{\sigma})^{-1} \sigma\right) \bar{\sigma} \in C(G / H)$. This is the identity on $H$, hence on $C$, and is an isomorphism of $R$-modules if it is 1-1. But $\psi_{\sigma}\left(\Sigma_{i \in G} r_{i} \sigma_{i}\right)=0$ implies $\Sigma_{\sigma_{i} \in G} r_{i}\left(v\left(\bar{\sigma}_{i}\right)^{-1} \sigma_{i}\right) \bar{\sigma}_{i}=0$, so for each $\bar{\sigma}$ in $G / H, \Sigma r_{i}\left(v\left(\bar{\sigma}_{i}\right)^{-1} \sigma_{i}\right)=0$ where $\sigma_{i}$ runs through the coset of $\sigma \bmod H$. If we set $\sigma_{i}=\tau_{i} \sigma, \tau_{i} \in H$, then this sum becomes $\Sigma_{h \in H} r_{i}\left(v(\bar{\sigma})^{-1} \sigma \tau_{i}\right)=0$, i.e., $v(\bar{\sigma})^{-1} \sigma \Sigma r_{i} \tau_{i}=0$. But $v(\bar{\sigma})^{-1} \sigma \tau_{i}$ are distinct elements of $H$ for $\tau_{i} \in H$, so are linearly independent over $R$; hence, $r_{t}=0$. Thus $\psi$ is an isomorphism of $R$-modules. Define a product on $C(G / H)$, call it $C(G / H)_{h}$, to coincide with that on $R G_{g}$. Then $\psi$ yields an isomorphism of $C$-algebras and $C(G / H)_{h}$ is a commutative twisted group ring with twisting $h: G / H \times G / H \rightarrow C$. Then $A$ is a crossed product as described. (In fact, $A$ may be viewed as a smash product $S \#_{C} C[G / H]_{h}$ with respect to the $(G / H)_{t}$-action on $S$ and the $G / H$-grading on $C[G / H]_{h}$.)

Grade $A=\Delta\left(S,(G / H)_{t}\right)_{h}=R G_{g} \otimes_{C} S$ diagonally. To show that $S=$ $\pi(A)$ we must show $A^{A_{1}}=S, A^{A}={ }^{A} A=R$. First, $S \subseteq A^{A_{1}}$ : for if $s_{\sigma z^{-1}} \in A_{1}$,

$$
s s_{\sigma_{\sigma} z^{-1}}=\phi\left(\sigma^{-1}, s\right) s_{\sigma} s^{\sigma} z_{\sigma^{-1}}=\phi\left(\sigma^{-1}, s\right) s_{\sigma} \phi(\sigma, s) z_{\sigma^{-1}}\left(s^{\sigma}\right)^{\sigma^{-1}}=s_{\sigma_{\sigma^{-1}}} s .
$$

If $z_{\rho} \in A^{A}$ then

$$
\begin{aligned}
& z_{\rho} s_{\sigma} z_{\sigma^{-1}}=s_{\sigma^{2}}{ }_{\sigma^{-1}} z_{\rho}=s_{\sigma}{ }_{\rho} z_{\sigma^{-1}} \text { since } R G_{g} \text { is commutative } \\
& =\phi\left(\rho^{-1}, s_{\sigma}\right) z_{\rho} s_{\sigma_{\sigma} z^{-1}}
\end{aligned}
$$

so $s_{\sigma}^{\rho}=\phi\left(\rho, s_{\sigma}\right) s_{\sigma}$. Since $s_{\sigma}$ may be chosen at random from $h S, \rho \in H$, and so $z_{\rho} \in C \subseteq S$. Thus $A^{A_{1}}=S$. Now $A^{A} \subseteq A^{A_{1}}=S$. So 


$$
\begin{aligned}
A^{A} & \subseteq\left\{s \in S \mid z_{\sigma} s=\phi(\sigma, s) s z_{\sigma} \text { for all } \sigma \text { in } G\right\} \\
& =\left\{s \in S \mid z_{\sigma} s=\phi(\sigma, s) \phi\left(\sigma^{-1}, s\right) z_{\sigma^{s}} s^{\sigma}\right\} \\
& =\left\{s \in S \mid s=s^{\sigma}\right\}=R
\end{aligned}
$$

so $R=A^{A}$. Also ${ }^{A} A \subseteq A^{A_{1}}=S$, and a similar argument shows ${ }^{A} A \subseteq R$. Thus ${ }^{A} A=R$. $A$ exists.

We have therefore shown that whenever $C=R H_{f} \subseteq R G_{g}$, then such an

In case $G=H \times J, J=G / H$ and we can replace $R G_{g}=C\left((G / H)_{t}\right)_{h}$ by $C(J)_{1}$, the usual group ring of $J$. In that case it is not necessary to assume any conditions on $\operatorname{Pic}(R): A=\Delta(S, J)_{1}$.

The proof of the theorem is complete as soon as we prove

(4.4) LEMMA. Let $G$ be a finite abelian group which acts trivially on the abelian group $A$. If $H$ is a subgroup of $G$, then res: $H_{\mathrm{sym}}^{2}(G, A) \rightarrow$ $H_{\text {sy } \mathrm{m}}^{2}(H, A)$ is onto.

Proof: We shall write the operation on $A$ as addition. We denote by $H_{\text {sy m }}^{2}(G, A)$ the subgroup of $H^{2}(G, A)$ consisting of classes represented by symmetric cocycles, those cocycles $f: G \times G \rightarrow A$ satisfying $f(\sigma, \tau)=f(\tau, \sigma)$. Note that coboundaries are always symmetric. From [20, Theorem 2.1 and p. 159] we know that if $H=W_{1} \times \cdots \times W_{r}$, then

$$
H_{\mathrm{sy} \mathrm{m}}^{2}(H, A) \cong \bigoplus_{i=1}^{r} H_{\mathrm{sy} \mathrm{m}}^{2}\left(W_{i}, A\right)
$$

the isomorphism being induced by restriction. Similarly for $G$. Thus it suffices to prove the lemma assuming $H$ is cyclic of order $p^{f}=c$ ( $p$ prime) with generator $\tau$, and we can assume $G$ is a $p$-group.

Let $G=Z_{1} \times \cdots \times Z_{n}$ where $Z_{i}=\left\langle\sigma_{i}\right\rangle$ is cyclic of order $d_{i}=p^{e_{i}}$. Let $\tau=\sigma_{1}^{r_{1}} \cdots \sigma_{n}^{r_{n}}$. If $\tau$ has order $p_{r_{i}}^{f}$ then ${ }_{u r_{i}}^{r_{i}}$ has order exactly $p^{f}=c$ for some $i$. Fix such an $i$. Replacing $\sigma_{i}^{r_{i}}$ by $\sigma_{i}^{u r_{i}}$ where $u$ is some appropriate integer relatively prime to $p$, we can assume that $r_{i} c=d_{i}$. Now $A / c A \cong$ $H^{2}(H, A)$ : an isomorphism is given by sending the class of $a$ to the class of $f_{a, \tau}$, where, setting $[r]=$ the greatest integer $\leqslant r$,

$$
f_{a, \tau}\left(\tau^{\lambda}, \tau^{\mu}\right)=a[(\lambda+\mu) / c] .
$$

(That is, $f_{a, \tau}$ is the cup product of $a+c A$ in $\breve{H}^{0}(H, A)$ (Tate cohomology) with the class of $\delta \chi_{\tau}$ in $H^{2}(H, \mathrm{Z})$ where $\chi_{\tau}: H \rightarrow \mathrm{Q} / \mathrm{Z}$ is given by $\chi_{\tau}\left(\tau^{\lambda}\right)$ $=\lambda / n$-see $\left[16\right.$, p. 141] .) So it suffices to show that $f_{a, \tau}: H \times H \rightarrow A$ is 
the restriction of a 2-cocycle $g$ on $G$. We choose such a $g: G \times G \rightarrow A$ as follows:

$$
g\left(\sigma_{1}^{\alpha_{1}} \cdots \sigma_{n}^{\alpha_{n}}, \sigma_{1}^{\beta_{1}} \cdots \sigma_{n}^{\beta_{n}}\right)=g\left(\sigma_{i}^{\alpha_{i}}, \sigma_{i}^{\beta_{i}}\right)=a\left[\left(\alpha_{i}+\beta_{i}\right) / d_{i}\right] .
$$

Then

$$
\operatorname{res} g\left(\tau^{\lambda}, \tau^{\mu}\right)=g\left(\sigma_{i}^{r_{i} \lambda}, \sigma_{i}^{r_{i} \mu}\right)=a\left[\frac{r_{i} \lambda_{+} r_{i} \mu}{d_{i}}\right]=a\left[\frac{\lambda+\mu}{c}\right]=f_{a, \tau}\left(\tau^{\lambda}, \tau^{\mu}\right) .
$$

So res $g=f_{a, \tau}$. Also, $g$ is a 2-cocycle. For $g$ is a 2-cocycle iff $\operatorname{res}_{G \rightarrow Z_{i}} g$ is a cocycle in $H^{2}\left(Z_{i}, A\right)$, clearly; and $\operatorname{res}_{G \rightarrow Z_{i}} g=f_{a, \sigma_{i}}$ can be shown to be a cocycle either directly or by identifying it as the image of a cup product, as above. That completes the proof of (4.4) and (4.3).

5. Dimodule algebras. In this section we note the relationship between $B_{\phi}(R, G)$ and a Brauer group $B D(R, G)$ of $G$-dimodule algebras defined by F. W. Long [12], [13] and apply the results of the previous sections to $B D(R, G)$.

Assume $G$ is a finite abelian group, and $H=R G$. A $G$-dimodule algebra $A$ is a $G$-graded algebra and a $G$-module algebra such that the $G$-action preserves the grading, that is, the following diagram [13, Definition 3.1(i)] commutes:

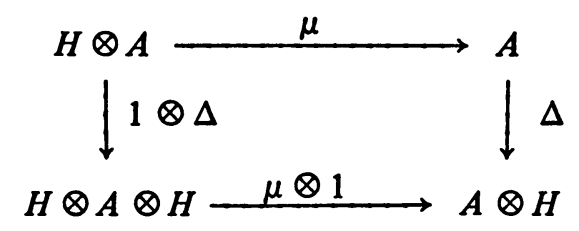

Let $H^{*}$ be the linear dual of $H$. We may define an $H^{*}$-module structure on $A$ from the $H$-grading on $A$ via the action $h^{*}(a)=\left(1 \otimes h^{*}\right) \Delta(a)$.

This $H^{*}$-module structure and the $H$-module structure commute, since they operate on different factors of $\Delta(a)$ for $a$ in $A$. Thus $A$ is an $H^{*} \otimes$ $H$-module. If $A$ is a $G$-graded algebra, then it is an $H$-comodule algebra, hence [13, Remark following Definition 2.9] an $H^{*}$-module algebra. Thus $A$ is an $H^{*} \otimes H$-module algebra. Thus $A$ is an $H \otimes H^{*}$-comodule algebra.

So an $H$-dimodule algebra is an $H \otimes H^{*}$-comodule algebra. The converse is equally clear.

Given two $G$-dimodule algebras $A$ and $B$ their product is the smash product $A \# B$, with multiplication given on homogeneous elements by

$$
\left(a_{1} \# b_{1}\right)\left(a_{2} \# b_{2}\right)=a_{1}^{b_{1}} a_{2} \# b_{1} b_{2} \text {, }
$$

where if $b_{1}$ is homogeneous of grade $\sigma,{ }^{b} a_{2}={ }^{\sigma} a_{2}$. In terms of the $(R G)^{*}$. comodule structure of $A$, 


$$
{ }^{a} a=\sum_{(a)}\left\langle\sigma, a_{(2)}\right\rangle a_{(1)} .
$$

Suppose the order of $G$ is a unit in $R$, and $\operatorname{Hom}(G, U(R))=G^{*} \cong G$, so that $(R G)^{*}=R\left(G^{*}\right)$. Then $A=\Sigma_{\chi \in G^{*}} A_{\chi}$ such that, for $a \in A_{\chi}, \Delta(a)=$ $a \otimes u_{\chi}$, and the smash product on homogeneous elements becomes

$$
\left(a_{1} \# b_{1}\right)\left(a_{2} \# b_{2}\right)=a_{1} a_{2}\left\langle G-\operatorname{grade}\left(b_{1}\right), G^{*}-\operatorname{grade}\left(a_{2}\right)\right\rangle \# b_{1} b_{2} \text {. }
$$

Let $\phi:\left(G \times G^{*}\right) \times\left(G \times G^{*}\right) \rightarrow U(R)$ be the bilinear map defined by

$$
\phi\left(\left(\sigma_{1}, \chi_{1}\right),\left(\sigma_{2}, \chi_{2}\right)\right)=\left\langle\sigma_{1}, \chi_{2}\right\rangle
$$

Then $A \# B=A \otimes B$, an instance of the graded tensor product of [CGO].

It is easy to check that $G$-Azumaya in the sense of Long and graded Azumaya in the sense of [CGO] coincide by observing that the maps $F, G$ of [12, Definition following Theorem 1.3] coincide with the maps $\eta_{A}, \mu_{A}$ of [CGO, (2.8)].

Since the Azumaya algebras which are trivial in [CGO, (2.10)] and in [12, Definition preceding Theorem 1.5] coincide, we have, summing up:

(5.1) THEOREM. If $R$ is a connected commutative ring, $G$ is an abelian group of order $n$ and exponent $m$, and $R$ contains $1 / n$ and a primitive mth root of unity, then Long's Brauer group $B D(R, G)$ is equal to $B_{\phi}\left(R, G \times G^{*}\right)$ $\cong B_{\phi}(R, G \times G)$ for $\phi$ as above.

We note that when $G$ is a $p$-group whose $r$ cyclic direct summands all have equal order, then $G \times G$ is also, and the matrix $M$ arising in $\S 3$ corresponding to the $\phi$ of the theorem is nonsingular-it is $\left(\begin{array}{ll}0 & I \\ I & 0\end{array}\right)$ where $I$ is the $r \times r$ identity matrix.

Summing up our results as applied to Long's group $B D(R, G)$ we have

(5.2) THEOREM. If $G=\Pi_{p}\left(\Pi_{i=1}^{r} Z_{p}{ }_{e_{p}}\right)$ has order $n$, and $R$ is a connected commutative ring containing $1 / n$ and a primitive $p^{e}$ th root of unity for each $p \mid n$ and $\operatorname{Pic}(R)$ is p-torsion free for all $p \mid n$, then $B D(R, G)$ is described by the two exact sequences

$$
\begin{gathered}
0 \rightarrow B(R) \rightarrow B D(R, G) \stackrel{\pi}{\longrightarrow} \operatorname{Galz}_{\phi}(R, G \times G) \rightarrow 0, \\
0 \rightarrow \operatorname{Comm}(R, G \times G) \rightarrow \operatorname{Galz}_{\phi}(R, G \times G) \stackrel{\beta}{\longrightarrow} \prod_{p} o_{Z / p^{e} Z}^{2 r_{p}}\left(\begin{array}{ll}
0 & I \\
I & 0
\end{array}\right),
\end{gathered}
$$

where $\beta$ is onto $O_{Z / p^{e}}^{2 r_{p}}\left(\begin{array}{ll}0 & I \\ I & 0\end{array}\right)$ for $p$ odd, and the image of $\beta$ in $O_{Z / 2}^{2 r_{2}} r_{I}\left(\begin{array}{ll}0 & I\end{array}\right)$ is 


$$
\left\{P \mid\left[(I-P)\left(\begin{array}{ll}
0 & 0 \\
I & 0
\end{array}\right)\left(I-P^{\prime}\right)-(I-P)\left(\begin{array}{ll}
0 & I \\
I & 0
\end{array}\right)\right]_{i i}=0 \text { for all } i=1, \cdots, 2 r_{2}\right\} \text {. }
$$

The assumption that $\operatorname{Pic}(R)$ is p-torsion free may be omitted if $r_{p}=1$ and $p$ is odd, or if $e_{p}=1$.

Proof. All but the last statement follows from what we have already done.

If $e_{p}=1$ then $G_{p}=\Pi_{i=1}^{r} Z_{p}$ and every subgroup is a direct summand, so by Theorem (4.3) the assumption on $\operatorname{Pic}(R)$ is not needed.

If $r_{p}=1$ and $p$ is odd the $p$ part of the theorem states that for $G=$ $Z_{p} e$

$$
0 \rightarrow \operatorname{Comm}(R, G \times G) \rightarrow \operatorname{Galz}_{\phi}(R, G \times G) \rightarrow O_{Z}^{2}{ }_{p}\left(\begin{array}{ll}
0 & 1 \\
1 & 0
\end{array}\right) \rightarrow 0
$$

is exact.

We show $\pi$ is onto by showing that for each $S$ in $\operatorname{Galz}(R, G \times G)$, the gradings $H$ of the center are a direct summand of $G \times G$. We do this by explicitly computing $O_{Z / p^{e} Z}^{2}\left(\begin{array}{ll}0 & 1 \\ 1 & 0\end{array}\right)$.

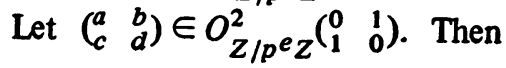

$$
\left(\begin{array}{ll}
a & b \\
c & d
\end{array}\right)\left(\begin{array}{ll}
0 & 1 \\
1 & 0
\end{array}\right)\left(\begin{array}{ll}
a & c \\
b & d
\end{array}\right)=\left(\begin{array}{ll}
0 & 1 \\
1 & 0
\end{array}\right)
$$

so $2 a b=0=2 c d, a d+b c=1$. From $a b=0$, we get, in $Z, a=u p^{r}, b=$ $v p^{s}$ with $r+s \geqslant e,(u v, p)=1$. But from $a d+b c=1$, either $r=0$ or $s=0$. Similarly for $c$ and $d$. So we have only two cases.

Case 1. $a=u, d=u^{-1}, c=b=0$ in $\mathrm{Z} / p^{e} \mathrm{Z}$.

Case 2. $b=u, c=u^{-1}, a=d=0$. We use the notation of $\$ 3$.

Case 1.

$$
P=\left(\begin{array}{cc}
u & 0 \\
0 & 1 / u
\end{array}\right), \quad A=\left(\begin{array}{cc}
0 & 1-u \\
1-1 / u & 0
\end{array}\right), \quad D=\left(\begin{array}{cc}
0 & u-1 \\
1-u & 0
\end{array}\right)=A\left(\begin{array}{cc}
-u & 0 \\
0 & -1
\end{array}\right) \text {. }
$$

The center is

$$
\left\{r A \mid r \in Z_{p^{e}}^{2}, r D=0\right\}=\left\{r A \mid r A\left(\begin{array}{rr}
u & 0 \\
0 & -1
\end{array}\right)=0\right\}=\{0\}
$$

since $\left(\begin{array}{ll}u & 0 \\ 0 & 1\end{array}\right)$ is invertible.

Case 2.

$$
P=\left(\begin{array}{cc}
0 & u \\
1 / u & 0
\end{array}\right), \quad A=\left(\begin{array}{cc}
-u & 1 \\
1 & -1 / u
\end{array}\right), \quad D=0
$$


So the center is $\left\{r A \mid r \in Z_{p^{e}}^{2}\right\}=$ the row space of $A$. But the row space of $A$ is isomorphic to $Z_{p}$, so is a direct summand of $G \times G$. That proves the case $r_{p}=1, p$ odd.

(5.3) REMARK. Observe that in this last proof we obtained essentially that

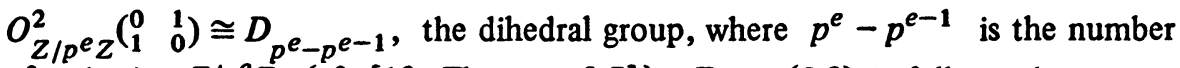
of units in $Z / p^{e} Z$ (cf. [12, Theorem 2.7]). From (5.2) it follows that $B D\left(C, Z / p^{e} Z\right) \cong D_{p^{e}-p^{e-1}}$. The complexity of $O_{Z / p^{e} Z}^{2_{r}}$, and hence of $B D\left(\mathrm{C},\left(Z / p^{e} Z\right)^{r}\right)$ is much greater if $r_{p}>1$. For example, for $p=3, n=2$, $e=2$, there is a short exact sequence

$$
0 \rightarrow(Z / 3 Z)^{6} \rightarrow O_{Z / 9 Z}^{4}\left(\begin{array}{ll}
0 & I \\
I & 0
\end{array}\right) \rightarrow O_{Z / 3 Z}^{4}\left(\begin{array}{ll}
0 & I \\
I & 0
\end{array}\right) \rightarrow 0
$$

$O_{Z / 3 Z}^{4}\left(\begin{array}{ll}0 & I \\ I & 0\end{array}\right)$ has a subquotient isomorphic to $P S L_{2}(Z / 3 Z) \times P S L_{2}(Z / 3 Z)$ [1] and the whole group has order $3^{8} \times 2^{7}$. When the target of $\pi$ is so complicated a direct approach to showing that $\pi$ is onto (as used in the proof of the last statement of (5.2)) has proved unfruitful.

\section{REFERENCES}

1. E. Artin, Geometric algebra, Interscience, New York, 1957. MR 18, 553.

2. M. Auslander and $O$. Goldman, The Brauer group of a commutative ring, Trans. Amer. Math. Soc. 97 (1960), 367-409. MR 22 \#12130.

3. H. Bass, Lectures on topics in algebraic $K$-theory, Tata Inst. Fund. Res. Lectures on Math., no. 41, Tata Institute of Fundamental Research, Bombay, 1967. MR 43 \#4885.

4. S. U. Chase, D. K. Harrison and A. Rosenberg, Galois theory and Galois cohomology of commutative rings, Mem. Amer. Math. Soc. No. 52 (1965), 15-33. MR 33 \#4118.

5. L. N. Childs, G. S. Garfinkel and M. Orzech, The Brauer group of graded Azumaya algebras, Trans. Amer. Math. Soc. 175 (1973), 299-326.

6. L. N. Childs, Abelian Galois extensions of rings contatning roots of unity, Illinois J. Math. 15 (1971), 273-280. MR 43 \#287.

7. - A note on the fixed ring of a Galois extension, Osaka J. Math. 4 (1967), 173-176. MR 36 \#1490.

8. F. R. DeMeyer, Galois theory in separable algebras over commutative rings, Illinois J. Math. 10 (1966), 287-295. MR 33 \#149.

9. A. Fröhlich and C. T. C. Wall, Generalizations of the Brauer group. I (to appear).

10. D. K. Harrison, Abelian extensions of commutative rings, Mem. Amer. Math. Soc. No. 52 (1965), 1-14. MR 33 \#4117.

.11. T. Kanzaki, On Galois algebra over a commutative ring, Osaka J. Math. 2 (1965), 309-317. MR 33 \#150.

12. F. W. Long, A generalization of the Brauer group of graded algebras, Proc. London Math. Soc. (3) 29 (1974), 237-256.

13. - The Brauer group of dimodule algebras, J. Algebra 30 (1974), 559-601.

14. D. J. Picco and M. I. Platzeck, Graded algebras and Galois extensions, Rev. Un. Mat. Argentina 25 (1971), 401-415.

15. A. Rosenberg and D. Zelinsky, Automorphisms of separable algebras, Pacific J. Math. 11 (1961), 1109-1117. MR 26 \#6215.

16. J.-P. Serre, Corps locaux, Publ. Inst. Mathématique Univ. Nancago, VIII, Actu- 
alités Sci. Indust., no. 1296, Hermann, Paris, 1962. MR 27 \#133.

17. C. Small, The Brauer-Wall group of a commutative ring, Trans. Amer. Math. Soc. 156 (1971), 455-491. MR 43 \#1966.

18. O. E. Villamayor and D. Zelinsky, Galois theory with infinitely many idempotents, Nagoya Math. J. 35 (1969), 83-98. MR 39 \#5555.

19. C. T. C. Wall, Graded Brauer groups, J. Reine Angew. Math. 213 (1963/64), 187-199. MR 29 \#4771.

20. K. Yamazaki, On projective representations and ring extensions of finite groups, J. Fac. Sci. Univ. Tokyo Sect. I 10 (1964), 147-195. MR 31 \#482.

DEPARTMENT OF MATHEMATICS, STATE UNIVERSITY OF NEW YORK, ALBANY, NEW YORK 12222 